\title{
Functional Genetic Variation in Dopamine Signaling Moderates Prefrontal Cortical Activity During Risky Decision Making
}

\author{
Milky Kohno', Erika L Nurmi', Christopher P Laughlin', Angelica M Morales', Emma H Gail', \\ Gerhard S Hellemann' and Edythe D London ${ }^{*, 1,2,3}$
}

'Department of Psychiatry and Biobehavioral Sciences and Semel Institute, University of California Los Angeles, Los Angeles, CA, USA; ${ }^{2}$ Department of Molecular and Medical Pharmacology, University of California Los Angeles, Los Angeles, CA, USA; ${ }^{3}$ Brain Research Institute, David Geffen School of Medicine, University of California Los Angeles, Los Angeles, CA, USA

\begin{abstract}
Brain imaging has revealed links between prefrontal activity during risky decision-making and striatal dopamine receptors. Specifically, striatal dopamine D2-like receptor availability is correlated with risk-taking behavior and sensitivity of prefrontal activation to risk in the Balloon Analogue Risk Task (BART). The extent to which these associations, involving a single neurochemical measure, reflect more general effects of dopaminergic functioning on risky decision making, however, is unknown. Here, 65 healthy participants provided genotypes and performed the BART during functional magnetic resonance imaging. For each participant, dopamine function was assessed using a gene composite score combining known functional variation across five genes involved in dopaminergic signaling: DRD2, DRD3, DRD4, DATI, and COMT. The gene composite score was negatively related to dorsolateral prefrontal cortical function during risky decision making, and nonlinearly related to earnings on the task. Iterative permutations of all possible allelic variations (7777 allelic combinations) was tested on brain function in an independently defined region of the prefrontal cortex and confirmed empirical validity of the composite score, which yielded stronger association than $95 \%$ of all other possible combinations. The gene composite score also accounted for a greater proportion of variability in neural and behavioral measures than the independent effects of each gene variant, indicating that the combined effects of functional dopamine pathway genes can provide a robust assessment, presumably reflecting the cumulative and potentially interactive effects on brain function. Our findings support the view that the links between dopaminergic signaling, prefrontal function, and decision making vary as a function of dopamine signaling capacity.
\end{abstract}

Neuropsychopharmacology (2016) 4I, 695-703; doi:I0.1038/npp.2015.192; published online 5 August 20I5

\section{INTRODUCTION}

Decision making is a complex executive function, and choices are often made in dynamic situations that require evaluation of potential risk and reward. Evidence from rodent studies has suggested that striatal dopaminergic neurotransmission contributes to risky decision making. For example, cue-evoked dopamine release in the nucleus accumbens core signals the preference for selecting either safe or risky rewards (Sugam et al, 2012), and pharmacological manipulations that increase the activation of D1 and D2 receptors bias choice toward larger, probabilistic rewards, whereas D3 receptors appear to exert opposing effects on this type of risk-based decision making (St Onge and Floresco, 2009). Current research findings point to a distributed network involving the frontal lobes in decision making. Human neuroimaging studies have indicated that there is a sequential

\footnotetext{
*Correspondence: Dr ED London, Semel Institute of Neuroscience and Human Behavior, University of California Los Angeles, 760 Westwood Plaza, Los Angeles, CA 90024-1759, USA, Tel: +310 825 0606, Fax: +3108250812, E-mail: elondon@mednet.ucla.edu

Received 9 February 2015; revised 2 June 2015; accepted 3 June 20 I5; accepted article preview online 29 June 2015
}

involvement of different brain regions in distinct stages of decision making (Ernst and Paulus, 2005), with elevated probabilities of activation in frontal and parietal cortex, thalamus, and caudate during ambiguous or risky decision making (Krain et al, 2006). Risk taking is also associated with activation in prefrontal cortex, insula, midbrain, and striatum, and sensitivity of dorsolateral prefrontal cortex (DLPFC) activation to risk during decision making is linked to striatal D2-type (D2 and D3) dopamine receptor availability (BPND) (Kohno et al, 2013) and to resting-state functional connectivity between the DLPFC and striatum (Kohno et al, 2014).

Involvement of dopaminergic neurotransmission has been hypothesized to underlie individual variation in risky decision making, an important phenotypic target in several neuropsychiatric disorders (Rahman et al, 2001). Genetic composite scores have been used as an index for dopaminergic activity, and a link between genes involved in dopamine signaling capacity and ventral striatal reactivity to rewards has been demonstrated (Nikolova et al, 2011; Stice et al, 2012). Because neuropsychiatric disorders that feature decisionmaking deficits are associated with dysregulated dopamine function (Frank et al, 2007; Rahman et al, 2001), 
Table I Genetic Variants Included in the Composite Score and their Effects on Dopaminergic Function

\begin{tabular}{lcl}
\hline Gene variant & Genotype & Effects on striatal dopaminergic function \\
\hline DRD2 rs2283265 & G/T & $\begin{array}{l}\text { Intronic SNP in DRD2 moderates alternative splicing of exon 6, influencing the proportion of dopamine D2 } \\
\text { long (D2L) and D2 short (D2S) receptor expression. }\end{array}$
\end{tabular}

D2S receptors are presynaptic, located on mesencephalic and corticostriatal projection axons (Khan et al, 1998). D2L receptors are predominantly located postsynaptically on striatal GABAergic neurons (Khan et al, 1998; Usiello et al, 2000). Presence of the T-allele favors D2L over D2S expression, and this potentiates corticostriatal glutamate release (De Mei et al, 2009) leading to the activation of striatal GABAergic medium spiny neurons. The result is the inhibition of striatal DA release through the inhibition of midbrain dopamine neurons (Centonze et al, 2003; Cepeda et al, 200 I).

TT and GT genotypes, score =0; GG homozygotes, score = I

DRD3 rs6280

Ser9Gly

Nonsynonymous SNP in DRD3 results in serine (ser) to glycine (gly) substitution and enhanced dopamine D3 receptor affinity.

D3 receptors, expressed on mesolimbic dopaminergic terminals, function in downregulating DA transmission. Compared with the ser allele, the gly allele is associated with greater striatal DA release, presumably through the effects on prostaglandin E2 production. Activation of EPI receptors on GABAergic efferents by prostaglandin E2 potentiates inhibitory synaptic input to midbrain, resulting in the inhibition of striatal DA release. The gly variant has been associated with less prostaglandin E2 production (Hellstrand et al, 2004) and greater reward-related DA release than the ser variant (Savitz et al, 20l3). ser/ser genotype, score =0; ser/gly and gly/gly, score $=$ I

DRD4 48-bp VNTR 2-II repeat VNTR repeat number, encoding a I6-amino acid segment in the third cytoplasmic loop of the dopamine D4 receptor, affects ability of the D4 dopamine receptor to heteromerize with D2S receptors.

D4 receptors on corticostriatal terminals contribute to regulating glutamate release and thereby striatal DA release (Lauzon and Laviolette, 20I0). DRD4 contains a polymorphic repeat in the third exon, most frequently consisting of 2, 4, or 7 repeats (D4.2, D4.4, and D4.7; Van Tol et al, 1992). Receptors containing 2 or 4 repeats form heteromers with D2-S isoforms; co-activation of D2S and DRD4.2 or DRD4.4 isoforms inhibits glutamate release from corticostriatal afferents, while the DRD4.7 variant does not dimerize with D2S receptors, and its expression may enhance glutamatergic neurotransmission and attenuate striatal DA release (Gonzalez et al, 20 I 2). 7-repeat carriers (one or two 7-alleles), score $=0$; all other variants, score $=1$

\section{DAT I SLC6A3 2-II repeat VNTR repeat number in the 3'UTR of DATI affects transporter expression.}

A meta-analysis of twelve studies, including 51 I subjects, indicated that presence of the 9R allele results in greater DAT expression (Faraone et al, 20 I3). $9 / 9$ and $9 / 10$ genotypes, score $=0 ; 10 / 10$ homozygotes, score $=1$

COMT rs 4680 Vall58Met Nonsynonymous SNP in COMT results in valine (val) to methionine (met) substitution, with reduced thermostability of the enzyme.

Compared with the met allele, the val allele has been associated with greater tyrosine hydroxylase gene expression in the substantia nigra (Akil et al, 2003) and greater dopamine synthesis rate, inferred from [F-I8]fluorodopa uptake (Meyer-Lindenberg et al, 2005). The val allele has also been related to greater disinhibition of mesencephalic DA activity than the met allele, through lower prefrontal cortical DA signaling and greater intrasynaptic levels of phasic striatal DA release (Bilder et al, 2004).

Scores of $0,0.5$, and I were assigned to met/met, val/met, and val/val, respectively.

understanding the link between markers for striatal dopamine activity and neural function in healthy individuals may help select therapeutic targets for pathological conditions. The goal of this study, therefore, was to evaluate how variability in striatal dopamine signaling, assessed using a gene composite score of known functional polymorphisms, affects brain function during risky decision making.

\section{Functional Variation in Dopamine Signaling Pathways}

A gene composite score was calculated for each participant to test the cumulative impact of functional variation across five genes purported to influence dopaminergic function. This score represented functional variation across genes encoding D2, D3, and D4 dopamine receptors (DRD2 rs2283265, $D R D 3$ rs6280, $D R D 4$ exon 3 variable number tandem repeat (VNTR), the dopamine transporter (DAT1 or SLC6A3) $3^{\prime}$-UTR VNTR) and the enzyme catechol-O-methyltransferase (COMT rs4680), involved in dopamine catabolism. The five genes were chosen a priori for inclusion in the composite score. Selection was on the basis of literature indicating effects of the functional variants on receptor or transporter expression and genetic association with risk-taking behavior or dopamine function in humans, and that orthologous genes affect dopamine signaling in animals. Variants were scored on the basis of literature regarding their contributions to striatal dopaminergic activity (see Table 1 for relevant references and scoring method).

\section{Risky Decision Making}

The Balloon Analogue Risk Task (BART), a test of risky decision making, presents a series of sequential choices, in which the participant decides either to pump a virtual balloon to increase potential gains while risking loss if the balloon explodes, or to cash out to retain earnings accrued on the trial. Previous studies have shown that the sensitivity of DLPFC activation to levels of risk during risky decision making on the BART is positively related to total earnings and to the functional connectivity of the corticostriatal pathway (Kohno et al, 2014), but negatively related to striatal D2-type receptor availability (Kohno et al, 2013). Based on these findings, we hypothesized that dopaminergic function, as determined by the gene composite score, would be negatively related to the sensitivity of activation in the 
DLPFC to levels of risk and reward, and negatively related to total earnings on the BART.

\section{MATERIALS AND METHODS}

\section{Participants}

Sixty-five healthy, right-handed participants (17-54 years of age) were recruited for this study, which was approved by the UCLA Office of the Human Research Protection Program. The sample comprised of 28 female and 37 male participants, of whom 26 reported Caucasian, 6 African American, 17 Hispanic, 15 Asian, and 1 Native American ancestry (Table 2). The exclusion criteria, determined on the basis of a physician-conducted history and physical examination and psychiatric evaluation using the Structured Clinical Interview for DSM-IV, were: systemic, neurological, cardiovascular, or pulmonary disease; head trauma with loss of consciousness; any current Axis I psychiatric diagnoses except nicotine dependence, and current use of prescribed psychotropic medications. Any participants who tested positive for cocaine, marijuana, methamphetamine, benzodiazepines, or opiates by urine toxicology were excluded, as were participants with MRI contraindications. Upon completing the study, participants were compensated in cash for their time and their performance on the BART. Of the 65 participants in the sample studied here, 13 were included in a study of D2-type dopamine receptor binding and fMRI during performance of the BART (Kohno et al, 2013), and 27 were included in a study of the relationship of resting-state functional connectivity to BART performance (Kohno et al, 2014).

\section{Genotyping}

Genomic DNA was extracted from whole blood using Qiagen's QIAamp protocol (Valencia, CA). Single-nucleotide polymorphisms in $D R D 2, D R D 3$, and COMT were genotyped by Life Technologies' TaqMan Allelic Discrimination Assay according to the manufacturer's protocols. The DAT1 (SLC6A3) $3^{\prime}$-UTR repeat and DRD4-exon 3 VNTR polymorphisms were genotyped using published methods and primers (Cook et al, 1995; Shaikh et al, 1993), with a modification of the DRD4 primers (F- 5'-CTACCCTGCCCGCTCATG-3'; R- 5'-CCGGTGATCTTGGCACGC-3').

\section{Dopamine Gene Composite Score}

A score was calculated by summing risk alleles at functional polymorphic loci in five genes chosen a priori (DRD2, DRD3, $D R D 4, D A T 1$, and COMT), on the basis of their previously demonstrated influence on striatal dopamine function. Each research participant was thereby assigned a gene composite score, resulting in scores ranging from 0 to 5 . Variants associated with the inhibition of striatal dopamine release or promoting glutamate release from cortical afferents were assigned a score of 0 , whereas those contributing to the inhibition of glutamate release from cortical afferents or promoting elevated striatal dopamine release were assigned a score of 1 . In cases where there was evidence for an intermediate phenotype in heterozygotes, a score of 0.5 was assigned (see Table 1).
Table 2 Characteristics of Research Participants ${ }^{\mathrm{a}}$

\begin{tabular}{lc}
\hline Age (years) & $25.122 \pm 10.32$ \\
Sex (no. of male/female) & $37 / 28$ \\
& 29 \\
Tobacco use (no. of smokers) & $17.57 \pm 2.87$ \\
Days used in the last 30 days & \\
Alcohol use & $1.46 \pm 0.26$ \\
Days used in the last 30 days & \\
Marijuana use & \\
Days used in the last 30 days & $0.33 \pm 0.12$ \\
& \\
Ethnicity & 26 \\
Caucasian & 6 \\
African American & 17 \\
Hispanic & 15 \\
Asian & 1 \\
American Indian & \\
\hline
\end{tabular}

Where appropriate, data are shown as means \pm SEM.

\section{fMRI Scanning}

Imaging was performed at $3 \mathrm{~T}$ on a Siemens Magnetom Trio MRI system. A set of 302 functional, T2*-weighted, echoplanar images (EPI) were acquired (slice thickness $=4 \mathrm{~mm}$; 34 slices; repetition time $=2 \mathrm{~s}$; echo time $=30 \mathrm{~ms}$; flip angle $=90^{\circ} ;$ matrix $=64 \times 64$; field of view $=200 \mathrm{~mm}$ ). High-resolution, T2-weighted, matched-bandwidth and magnetization-prepared rapid-acquisition gradient echo (MPRAGE) scans were also acquired. The orientation for matched-bandwidth and EPI scans was oblique axial to maximize brain coverage and to optimize signal from ventromedial prefrontal regions.

\section{Balloon Analog Risk Task}

A version of the BART, adapted for event-related fMRI, was used (Supplementary Figure 1). Balloons were either red or blue on active trials and white on control trials. When presented with an active balloon, participants selected between pumping the balloon for a potential increase in earnings ( $\$ 0.25 /$ pump) or cashing out to retain earnings accumulated during that trial. Pumping increased the size of the balloon and the accumulated earnings, or it resulted in balloon explosion and forfeiture of unrealized earnings accumulated during the trial. Trials began with the presentation of a balloon and included pumps on the given balloon and ended with the choice to cash out, which resulted in a display of the total earned for $2 \mathrm{~s}$, or in a balloon explosion, which was followed by a 2-s display of an exploded balloon with the message, 'Total $=\$ 0.00$.' Prior to scanning, participants were informed that red and blue balloons were associated with monetary reward and that they would receive their winnings after scanning, but they were not informed that the number of pumps to produce an explosion was pre-determined for each balloon from a 
uniform probability distribution (range of 1-8 for red balloons and 1-12 for blue balloons). Participants were informed that the white balloons did not explode and were not associated with potential reward, and were instructed to pump the white balloon until the trial ended. White control balloon trials were randomly interspersed among active trials, and the number of balloon presentations within the trial varied randomly between 1 and 12, according to a uniform distribution.

The task was administered in two 10-min runs. As each balloon remained on the screen until the participant pressed a button, the total number of completed trials varied by participant. The inter-stimulus interval for balloon presentations was randomly sampled from a uniform distribution ranging from 1-3 s, and the inter-trial interval was randomly sampled from an exponential distribution.

\section{Image Analysis}

Image analysis was performed using the FMRIB Software Library (FSL). The image series from each participant was first realigned to compensate for small head movements, and then a high-pass temporal filter (100 s) was applied. Data were spatially smoothed using a 5-mm FWHM Gaussian kernel and skull-stripped with the FSL Brain Extraction Tool. Registration was conducted through a three-step procedure, whereby EPI images were first registered to the matchedbandwidth image, then to the high-resolution MPRAGE structural image, and finally into standard Montreal Neurological Institute space, using 12-parameter affine transformations. Registration of MPRAGE structural images to standard space was further refined using FNIRT nonlinear registration. Statistical analyses were performed on data in native space using FMRIB's fMRI Expert Analysis Tool (FEAT), and the statistical maps were spatially normalized to standard space prior to higher-level analysis.

Four types of events were included in the general linear model (GLM): pumps on active balloons, cash outs, balloon explosions, and pumps on control balloons. Active-balloon and control-balloon pump events were defined as starting with the onset of the balloon presentation and ending with the button press to pump. Cash-out events were defined as the time between the appearance of the balloon and the disappearance of the feedback message giving the total earned. Balloon explosion events started with the appearance of the exploded balloon and ended with the message 'Total Earned $=\$ 0.00$ '. As a trial progressed, risk and potential reward increased with each pump, as did the amount earned with the choice to cash out. For each of the four types of events, estimates of mean activation and of parametric modulation of activation by pump number were included in a GLM using FEAT. Parametric regressors tested the linear relationship between pump number and blood oxygen leveldependent signal, by using demeaned pump number (pump number minus mean number of pumps within each trial) as a parametric modulator with greater weight assigned to later pumps. For example, within a trial, the second pump event, with greater risk and also reward, was given greater weight than the first. The parametric modulator for cash-out and explode events was the number of pumps before the decision to cash out or before the balloon exploded, respectively. The nonparametric regressors were used to estimate the mean response for each event without consideration of the escalation of potential reward/loss within the trial.

Whole-brain statistical analyses, using a fixed-effects model, were conducted separately for each imaging run per participant, and again to combine contrast images across the two runs. For between-participant analyses, a mixed-effects analysis was conducted with the FMRIB Mixed Effects simple ordinary least squares module with gene composite scores as a covariate of interest and sex and age as nuisance covariates. Sex was also examined as a covariate of interest and an interaction of sex and gene score on the modulation of activation was tested. In addition, allele frequencies differ between racial groups and can lead to spurious genetic associations. We, therefore, included ethnicity as a nuisance covariate in a separate analysis, and also conducted an analysis including only participants of Caucasian and Hispanic ethnicities $(n=43)$, which share similar allele frequencies at the loci examined. Finally, because of the interaction of cigarette smoking with the dopamine system (Brody et al, 2004, Le Foll et al, 2013), smoking status was added as a nuisance covariate in a separate analysis. All statistical images were thresholded at a voxel height of $\mathrm{Z}>2.3$ and a cluster-probability threshold of $p<0.05$, corrected for whole-brain multiple comparisons using the Theory of Gaussian Random Fields.

\section{Iterative Permutation Model}

In order to test the strength of the gene composite scoring system against other potential models, we first performed a linear regression against fMRI parameter estimates of the modulation of activation by risk in an independently selected region of interest. This analysis targeted the right middle frontal gyrus, which was associated with parametric modulation of activation with striatal D2-like receptor availability in our prior published findings (Kohno et al, 2013). The right middle frontal gyrus was sampled as a spherical ROI with a $10-\mathrm{mm}$ radius around the peak voxel (MNI coordinates: $x=30, y=36, z=20$ ) from a cluster previously associated with risk-taking on the BART (Rao et al, 2008). We examined the relationship between the modulation of activation by risk in this region of interest with all other possible scores derived from variants in the five genes considered. Specifically, scores for dosage/genotypic (1, 0.5, $0)$, dominant $(1,1,0)$, and recessive $(1,0,0)$ models along with their inverses (common $v s$ minor allele-governed) were evaluated, yielding six alternate combinations of variants, each permuted across the five genes for a total of 7776 tests. Results for each permutation, along with that of the original composite gene score, were percent ranked by $p$-value over the 7777 regressions. For comparison purposes, and as negative controls, arbitrary anatomical regions of interest (precuneus and visual cortex) as defined by the Harvard Oxford Cortical Atlas were tested.

\section{Behavioral Analysis}

A linear and curvilinear regression was used to examine the relationship between the gene composite score and performance on the BART using the Statistical Package for the Social Sciences version 21. In order to index adaptive decision making, total earnings were modeled as the dependent 


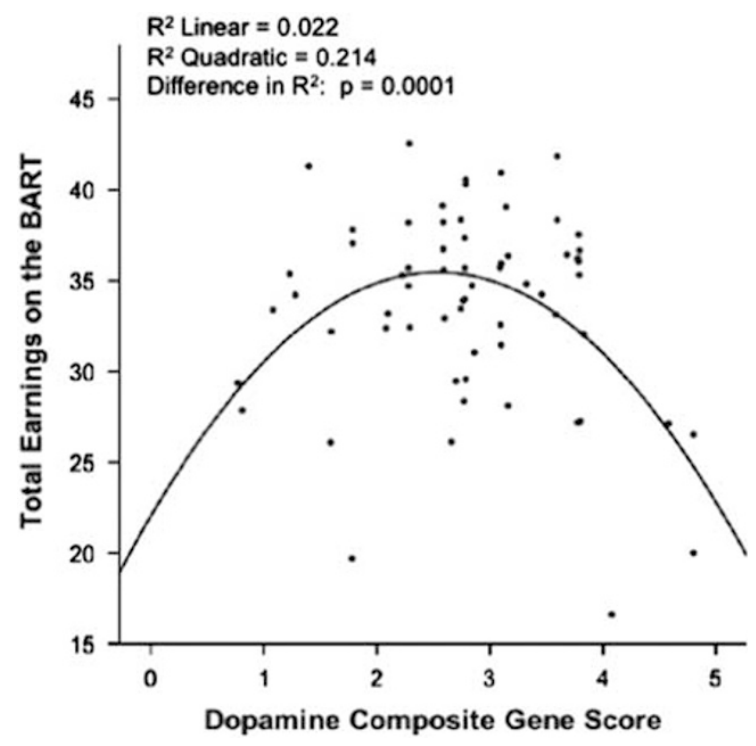

Figure I Nonlinear relationship between dopamine gene scores and earnings on the BART. Scatter plot depicts a quadratic relationship between dopamine gene scores and earnings on the BART. Values for earnings and dopamine composite gene scores were adjusted for age and sex.

variable with gene composite scores as the independent variable and age and sex as covariates. In a separate analysis, the number of pumps was modeled as the dependent variable. In addition, an $R^{2}$ difference test was conducted to determine whether including the curvilinear component significantly improved model fit.

\section{RESULTS}

\section{Gene Composite Score and Behavioral Performance}

Across subjects, gene composite scores (range: 0-5) were normally distributed (Supplementary Table 1). The gene composite score showed a quadratic relationship with total earnings on the BART. The model including the curvilinear term explained significantly more of the variability in total earnings $\left(R^{2}=0.214\right)$ than the model including only a linear term $\left(R^{2}=0.023 ; R^{2}\right.$ difference $=0.194, \mathrm{~F}(1,62)=15.61$, $p=0.0001$; Figure 1). When examining the independent linear effects of genes on total earnings, there were no significant associations after controlling for multiple comparisons. There were no significant relationships between the number of balloon pumps and the gene composite scores or individual loci.

\section{Gene Composite Score and fMRI BART Analysis}

In a whole-brain analysis, the gene composite score was positively related to the modulation of activation in the midbrain $\left(p=3.64 \times 10^{-6}\right)$ but negatively related to the modulation of activation in the left $\left(p=7.12 \times 10^{-5}\right)$ and right middle frontal gyrus $\left(p=9.91 \times 10^{-5}\right)$, right parietal cortex and posterior cingulate cortex $(p=0.017$; whole-brain cluster level $p$-values; Figure 2). These results remained significant after controlling for smoking status. Similar results were found in the analyses that included ethnicity

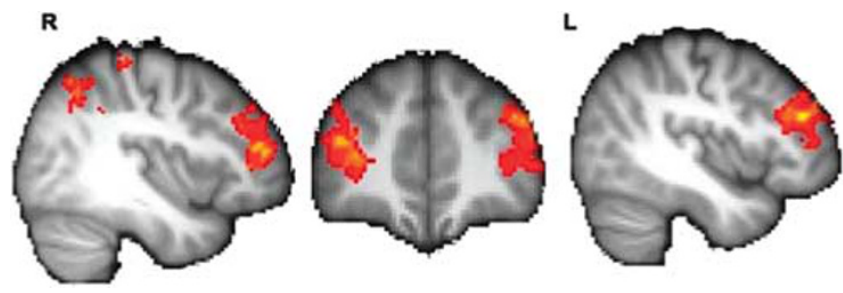

Figure 2 Negative relationship between dopamine gene scores and the parametric modulation of activation by pump number during risky decision making. Significant relationships were observed involving the right and left middle frontal gyrus, right parietal cortex, and posterior cingulate cortex $(p<0.05$, whole-brain cluster corrected, controlling for age and sex $(n=65))$. Statistical significance was retained, with the exception of the posterior cingulate cortex after excluding all but the Caucasian and Hispanic participants $(n=42)$ and after controlling for ethnicity $(n=65)$.

as a covariate and also when using only Caucasian and Hispanic participants $(n=43)$. In these analyses, activation in the right and left middle frontal gyrus and parietal cortex overlapped with the results obtained using the whole sample but there was no significant cluster of activation in the posterior cingulate cortex. There was a significant gene score by sex interaction on the modulation of activation in the left DLPFC. In post hoc analyses, the negative relationship between gene score and modulation of activation in the left DLPFC was stronger in men than in women. There were no significant interactive effects involving the right DLPFC. When examining independent effects of each gene on the cluster of activation in the right middle frontal gyrus, a significant negative relationship was found with DRD4 $(\beta=-19.451, p=0.002)$. No other significant independent effects were found; DRD2: $\beta=-9.479, p=0.124 ; D R D 3$ : $\beta=-13.072, p=0.120 ;$ DAT1: $\beta=1.015, p=0.877$; COMT: $\beta=-11.407, p=0.171)$. Although only DRD4 showed a significant negative relationship at $p<0.05$, a small effect was seen with DRD2 (Cohen's D=0.25), DRD3 (Cohen's $\mathrm{D}=0.24$ ) and COMT (Cohen's $\mathrm{D}=0.23$ ). There was no effect of DAT1 on the modulation of right middle frontal activation (Cohen's $\mathrm{D}=0.003$; Figure 3 ).

\section{Iterative Permutation of Gene Composite Scores and fMRI BART Analysis}

The data-driven permutation analysis found 7777 possible allelic combinations of the five genes of interest. When testing the predictive validity of these permutations on the modulation of activation in an independent functional right middle frontal gyrus region of interest (Kohno et al, 2013), the hypothesis-driven model ranked in the top 5\% $(p=0.018)$. There were no significant relationships between the gene composite score and the visual cortex or precuneus, which served as negative controls.

\section{DISCUSSION}

The prefrontal cortex and striatum interact during the process of decision making, with dopamine signaling mediating-behavior in the context of relative risk and reward (Stopper et al, 2014). This study supports the view that 


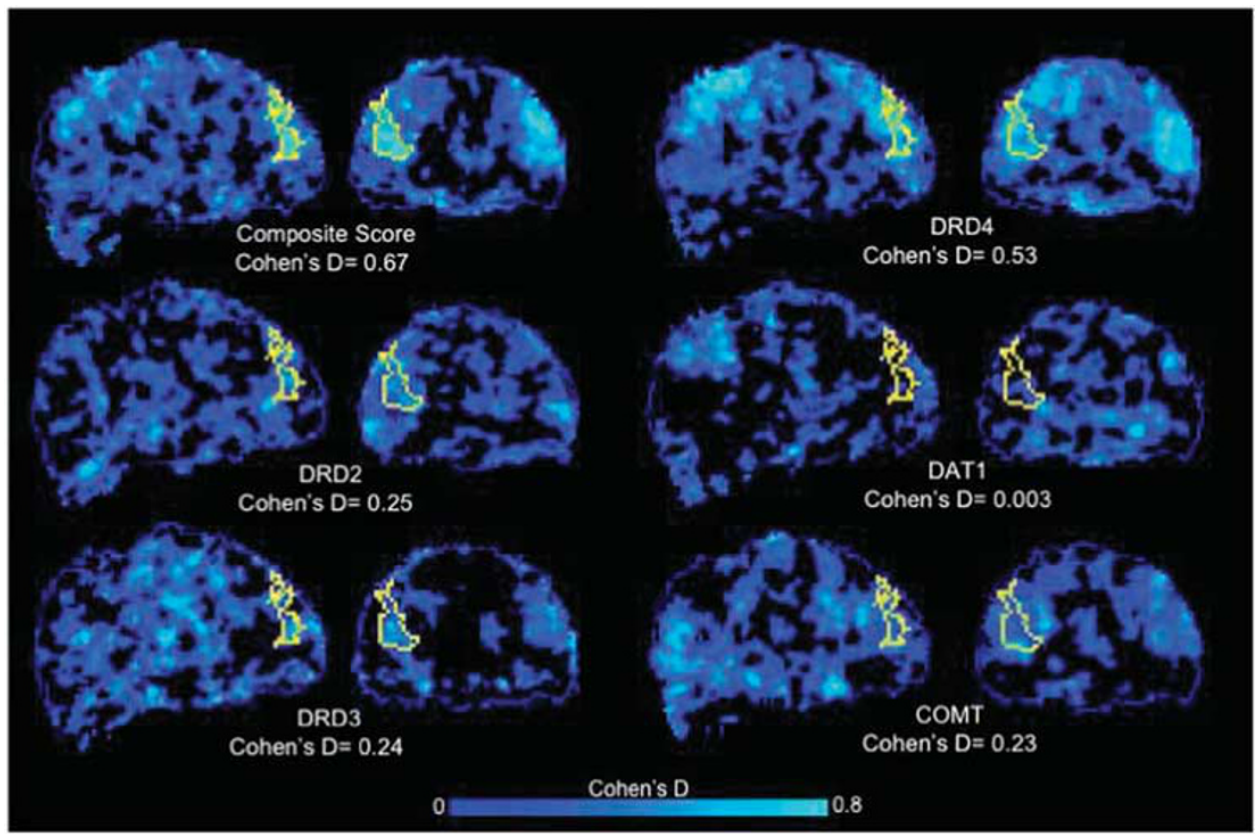

Figure 3 Effect size maps for the relationship between brain function during risky decision-making and dopamine gene score and each gene. For each gene, maps of effect sizes (Cohen's D) show the contribution of each gene to the parametric modulation of brain activation by risk during risky decision making. Outlined in yellow is the cluster in the right dorsolateral prefrontal cortex that showed a significant relationship with the dopamine gene score in the whole-brain analysis.

striatal dopaminergic signaling capacity (Cools, 2008), as indexed by composite scores presumably reflecting multilocus genetic effects, predicts prefrontal and midbrain activity during risky decision making. A growing literature suggests that dopaminergic abnormalities underlie impairments in decision making, which accompany a number of neuropsychiatric disorders (Rahman et al, 2001), but the effects of dopaminergic drugs on risk-taking behavior are inconsistent. For example, administration of dopamine agonists promotes risky choices in Parkinson's disease (Cools and D'Esposito, 2011) and in healthy controls (Riba et al, 2008), but reduces risk-taking behaviors (DeVito et al, 2008) and impulsive decisions (Shiels et al, 2009) in children with attention deficit hyperactivity disorder. Although the effects of amphetamine and haloperidol are not limited to dopaminergic systems, the administration of either drug, despite the opposite effects on dopamine signaling increases motivation to gamble in problem gamblers (Zack and Poulos, 2004, 2007), suggesting that the link between dopamine signaling and risk-taking behavior is not simply a linear relationship.

Notably, evidence from a variety of animal and human studies is consistent with an inverted U-shaped relationship between dopaminergic function and cognitive performance, perhaps depending on the variation in optimal dopamine levels in relevant brain regions. Along these lines, the effects of bromocriptine on working memory differ as a function of baseline dopamine synthesis capacity in healthy individuals, where low- or high-dopamine synthesis capacity is associated with improved or impaired performance, respectively (Cools et al, 2008). The observation, in the present study, of a quadratic relationship between gene composite scores and decision-making performance is also consistent with an inverted U-shaped relationship of dopaminergic function with behavior. Previous studies have examined the relationship between individual genes and performance on the BART. In one study, DAT1 10-repeat homozygotes exhibited greater risk-taking behavior than 9-repeat carriers (Mata et al, 2012). Another study showed that COMT-met homo-

zygotes took greater risks and subsequently earned more than val carriers (Lancaster et al, 2012). Here, we show that the number of balloon pumps were not significantly related to DAT1 and COMT genotypes or to the gene. The dopamine gene score, however, did account for $20 \%$ of the variance in overall earnings. The magnitude of this effect is notable given the potential contributions of nongenetic factors on risky decision making. The quadratic relationship suggests that low and high extremes of dopamine signaling reflect risk averse- and reward-seeking behavior, respectively. The lack of a relationship between the gene score and balloon pumps may be due to the limited sample size or reflect the fact that the number of balloon pumps can vary considerably from trial to trial.

As the midbrain is a region that is important in dopamine synthesis and release (Johnson et al, 1992), the positive relationship between gene composite scores and midbrain function is in line with the assumption that the gene composite score is an index for dopamine signaling. The negative relationship between modulation of DLPFC activation by risk during decision-making and the dopamine gene composite score extends the observation of a negative relationship between modulation of DLPFC function during risky decision making and striatal D2-like receptor availability (Kohno et al, 2013). The gene composite score includes genes that code for elements involved in mesocorticolimbic and corticostriatal signaling; therefore, the results provide insights into the dynamic interactions between frontal regions during decision-making and striatal dopamine signaling. Specifically, they support the notion that striatal 
dopamine signaling modulates top-down corticostriatal control to guide adaptive decision making (Dalley et al, 2011), as DLFPC activation was greater in participants with dopamine gene composite scores that presumably reflect less rather than more dopamine signaling.

Dopamine transmission can influence prefrontal activity through various signaling pathways including mesocortical projections that increase the effective threshold for striatal firing (Frank et al, 2001; Grace, 1991) and corticostriatal projections that influence glutamatergic synaptic transmission (Levine and Cepeda, 1998). These systems interact through an elaborate system involving dopamine receptors, transporters and catabolic enzymes that are important in maintaining the balance of striatal dopamine concentrations. Repeated firing of dopamine neurons induces a highamplitude increase in dopamine release within or near the synapse, and is thought to signal the presence of relevant stimuli. An increase in striatal dopamine signaling is regulated by actions on presynaptic and postsynaptic receptors and high-affinity dopamine transporters, while somatodendritic autoreceptors slow the firing rate of dopaminergic neurons and activation of nerve terminal autoreceptors inhibit the synthesis and release of dopamine (Dreyer and Hounsgaard, 2012; Meltzer, 1980). The firing of midbrain neurons is also mediated by presynaptic D2 receptors on the corticostriatal glutamate-containing projections from the prefrontal cortex to the ventral striatum (McGeer et al, 1977), and prefrontal dopaminergic activity also contributes to striatal dopamine release through descending midbrain and corticostriatal projections to attenuate phasic dopamine release in the ventral striatum (Grace, 1991).

Together, these key regulators of dopamine signaling maintain intrasynaptic dopamine homeostasis (Rudnick and Clark, 1993) and thereby likely contribute to goal-directed behavior. In line with this view, animal studies measuring dopamine efflux using microdialysis have shown contributions of the PFC and striatum in integrating reward, delay and uncertainty signals (St Onge et al, 2012), and pharmacological disruption of dopamine signaling produces effects on reward-driven behavior (Floresco et al, 2008). A role of striatal D2-type receptors in prefrontal cortical function has been inferred from rates of glucose metabolism (Volkow et al, 2001), and it has been proposed that abnormal activity of dopaminergic neurons may enhance stimulusreward associations (Wise, 2002) and the inhibition of reward-seeking behavior depends upon activity in corticostriatal projections (Jentsch and Taylor, 1999). The results of this study, therefore, underscore the influence of dopamine signaling on prefrontal predominance in behavioral control (Jentsch and Taylor, 1999). Together with observations that the modulation of DLPFC activation is related negatively to dopamine D2 receptor availability (Kohno et al, 2013) and positively to corticostriatal resting-state functional connectivity (Kohno et al, 2014), the results presented here provide further evidence that mesocorticolimbic and corticostriatal signaling mediates prefrontal brain function during decision making.

In concordance with our data, two prior reports found that an aggregate of genes provided a stronger account of the neural substrates underlying reward reactivity of the striatum than individual genes (Nikolova et al, 2011; Stice et al, 2012). Despite opposite scoring algorithms employed for DAT1 and
DRD4 genotypes in these two studies, both found that ventral striatal reactivity to food or monetary reward varied as a function of a multilocus genetic score. In these studies, polymorphisms were selected and scored on the basis of their presumed relevance to dopamine signaling. However, the contributions of these genes to dopaminergic signaling are incompletely understood; and theoretical gene scores are limited by assumptions of how allelic variants influence biological processes. The present study, therefore, incorporated a model-free, iterative, allelic scoring algorithm to examine the effects of every possible permutation of the five gene variant combinations. As model-free iterative methods are limited by data over fitting, the combination of both approaches provides an advantage by recognizing the limitations of each. In the case of this study, use of a robust iterative permutation test affirmed the relationship between the dopamine gene composite score and prefrontal brain function.

Similar to our findings of an interactive effect of sex and genotype on brain function, there is a recent report that COMT and DRD2 genotypes interact with sex to affect cognitive measures of attentional control (Gurvich and Rossell, 2015). Sexually dimorphic functioning of the mesolimbic dopamine system has been well documented in humans and animal models (Arvidsson et al, 2014; Kilford et al, 2014; Lippert et al, 2014; Uzefovsky et al, 2014). Women exhibit higher concentrations of extracellular dopamine (Riccardi et al, 2006), greater amphetamine-induced dopamine release (Riccardi et al, 2006), and higher levels of frontal cortical dopamine D2-type receptors (Kaasinen et al, 2001), and of striatal dopamine transporters (Staley et al, 2001) than men. Animal studies have shown that estrogen significantly increases striatal dopamine synthesis, baseline dopamine release, and d-amphetamine-induced dopamine release (Di Paolo, 1994), and studies suggest that these sex-related differences in dopamine function may explain sex differences in cognition and behavior (Gurvich and Rossell, 2015; Riccardi et al, 2006). Because neuropsychiatric disorders that feature decision-making deficits are associated with dysregulated dopamine function (Frank et al, 2007; Rahman et al, 2001) and show sex differences in the incidence, prevalence, and treatment outcome (Hartung et al, 2002), it is important to investigate how sex mediates the relationships between markers for striatal dopamine activity and brain function in future studies.

\section{Limitations}

Because genetic composite profiling is limited by the assumptions used in assigning the scores, a data-driven algorithm was implemented. Still the iterative permutations assumed an equal and additive weight for each genotype. In addition, the small sample size precluded direct examination of gene-gene interactions. In addition, although a positive relationship between modulation of striatal activation by risk and the dopamine gene score was expected, such a relationship was not found using the selected statistical criteria. At uncorrected thresholds, however, the gene score was positively related to modulation of activation in left ventral striatum $(Z$-score $=2.75, p=0.0029)$, right caudate $(Z$-score $=2.22, p=0.013)$, left putamen $(Z$-score $=2.18$, $p=0.015)$, and right pallidum $(Z$-score $=2.42, p=0.0077)$. 
Failure to find significance in these relationships may, in part, result from variability of striatal activation during risky decision making, possibly reflecting individual differences in risk tolerance and perception. Finally, while this study focused on functional polymorphisms involved in dopaminergic signaling, many more variants that were not included here undoubtedly contribute to the complex phenotype of risky decision making.

Despite these limitations, the findings of this study suggest that genetic variants with reported effects on striatal dopamine signaling influence decision making and the modulation of prefrontal cortical and midbrain activation to risk in healthy control subjects. The results suggest that brain function and risky decision making are linked to multiple markers of striatal dopamine activity. Future studies will be needed to determine the utility of a dopamine genetic score in refining and individually tailoring dopaminergic treatments for patients affected by dopamine-related disorders.

\section{FUNDING AND DISCLOSURE}

None of the authors have a financial relationship with any organization that sponsored this research. The authors declare no conflict of interest.

\section{ACKNOWLEDGMENTS}

This work was funded, in part, by a grant from Philip Morris, USA. Additional funding was provided by an endowment from the Thomas P and Katherine K Pike Chair in Addiction Studies and a gift from the Marjorie M Greene Trust. Dr AMM and Dr MK were supported by institutional training grant T32 DA 024635. None of the sponsors were involved with the design, collection, analysis or interpretation of data, writing the manuscript or the decision to submit the manuscript for publications.

\section{AUTHOR CONTRIBUTIONS}

Dr EDL, Principal Investigator, takes responsibility for the integrity of the data and the accuracy of the data analysis. All of the authors had full access to the data in the study. All authors have contributed to the scientific process leading up to the writing of this manuscript and to its preparation.

\section{REFERENCES}

Akil M, Kolachana BS, Rothmond DA, Hyde TM, Weinberger DR, Kleinman JE (2003). Catechol-O-methyltransferase genotype and dopamine regulation in the human brain. J Neurosci 23: 2008-2013.

Arvidsson E, Viereckel T, Mikulovic S, Wallen-Mackenzie A (2014). Age- and sex-dependence of dopamine release and capacity for recovery identified in the dorsal striatum of C57/B16J mice. PLoS One 9: e99592.

Bilder RM, Volavka J, Lachman HM, Grace AA (2004). The catechol-O-methyltransferase polymorphism: relations to the tonic-phasic dopamine hypothesis and neuropsychiatric phenotypes. Neuropsychopharmacology 29: 1943-1961.

Brody AL, Olmstead RE, London ED, Farahi J, Meyer JH, Grossman P et al (2004). Smoking-induced ventral striatum dopamine release. Am J Psychiatry 161: 1211-1218.
Centonze D, Gubellini P, Pisani A, Bernardi G, Calabresi P (2003). Dopamine, acetylcholine and nitric oxide systems interact to induce corticostriatal synaptic plasticity. Rev Neurosci 14: 207-216.

Cepeda C, Hurst RS, Altemus KL, Flores-Hernandez J, Calvert CR, Jokel ES et al (2001). Facilitated glutamatergic transmission in the striatum of D2 dopamine receptor-deficient mice. J Neurophysiol 85: 659-670.

Cook EH Jr, Stein MA, Krasowski MD, Cox NJ, Olkon DM, Kieffer JE et al (1995). Association of attention-deficit disorder and the dopamine transporter gene. Am J Hum Genet 56: 993-998.

Cools R (2008). Role of dopamine in the motivational and cognitive control of behavior. Neuroscientist 14: 381-395.

Cools R, D'Esposito M (2011). Inverted-U-shaped dopamine actions on human working memory and cognitive control. Biol Psychiatry 69: e113-e125.

Cools R, Gibbs SE, Miyakawa A, Jagust W, D'Esposito M (2008). Working memory capacity predicts dopamine synthesis capacity in the human striatum. J Neurosci 28: 1208-1212.

Dalley JW, Everitt BJ, Robbins TW (2011). Impulsivity, compulsivity, and top-down cognitive control. Neuron 69: 680-694.

De Mei C, Ramos M, Iitaka C, Borrelli E (2009). Getting specialized: presynaptic and postsynaptic dopamine D2 receptors. Curr Opin Pharmacol 9: 53-58.

DeVito EE, Blackwell AD, Kent L, Ersche KD, Clark L, Salmond CH et al (2008). The effects of methylphenidate on decision making in attention-deficit/hyperactivity disorder. Biol Psychiatry 64: 636-639.

Di Paolo T (1994). Modulation of brain dopamine transmission by sex steroids. Rev Neurosci 5: 27-41.

Dreyer JK, Hounsgaard J (2012). Mathematical model of dopamine autoreceptors and uptake inhibitors and their influence on tonic and phasic dopamine signaling. J Neurophysiol 109: 171-182.

Ernst M, Paulus MP (2005). Neurobiology of decision making: a selective review from a neurocognitive and clinical perspective. Biol Psychiatry 58: 597-604.

Faraone SV, Spencer TJ, Madras BK, Zhang-James Y, Biederman J (2013). Functional effects of dopamine transporter gene genotypes on in vivo dopamine transporter functioning: a metaanalysis. Mol Psychiatry 19: 880-889.

Floresco SB, Tse MT, Ghods-Sharifi S (2008). Dopaminergic and glutamatergic regulation of effort- and delay-based decision making. Neuropsychopharmacology 33: 1966-1979.

Frank MJ, Loughry B, O'Reilly RC (2001). Interactions between frontal cortex and basal ganglia in working memory: a computational model. Cogn Affect Behav Neurosci 1: 137-160.

Frank MJ, Scheres A, Sherman SJ (2007). Understanding decisionmaking deficits in neurological conditions: insights from models of natural action selection. Philos Trans R Soc Lond B Biol Sci 362: 1641-1654.

Gonzalez S, Rangel-Barajas C, Peper M, Lorenzo R, Moreno E, Ciruela F et al (2012). Dopamine D4 receptor, but not the ADHD-associated D4.7 variant, forms functional heteromers with the dopamine D2S receptor in the brain. Mol Psychiatry 17: 650-662.

Grace AA (1991). Phasic versus tonic dopamine release and the modulation of dopamine system responsivity: a hypothesis for the etiology of schizophrenia. Neuroscience 41: 1-24.

Gurvich C, Rossell SL (2015). Dopamine and cognitive control: sexby-genotype interactions influence the capacity to switch attention. Behav Brain Res 281: 96-101.

Hartung CM, Willcutt EG, Lahey BB, Pelham WE, Loney J, Stein MA et al (2002). Sex differences in young children who meet criteria for attention deficit hyperactivity disorder. J Clin Child Adolesc Psychol 31: 453-464.

Hellstrand M, Danielsen EA, Steen VM, Ekman A, Eriksson E, Nilsson CL (2004). The ser9gly SNP in the dopamine D3 receptor causes a shift from cAMP related to PGE2 related signal 
transduction mechanisms in transfected CHO cells. J Med Genet 41: $867-871$.

Jentsch JD, Taylor JR (1999). Impulsivity resulting from frontostriatal dysfunction in drug abuse: implications for the control of behavior by reward-related stimuli. Psychopharmacology (Berl) 146: 373-390.

Johnson SW, Seutin V, North RA (1992). Burst firing in dopamine neurons induced by $\mathrm{N}$-methyl-D-aspartate: role of electrogenic sodium pump. Science 258: 665-667.

Kaasinen V, Nagren K, Hietala J, Farde L, Rinne JO (2001). Sex differences in extrastriatal dopamine $\mathrm{d}(2)$-like receptors in the human brain. Am J Psychiatry 158: 308-311.

Khan ZU, Mrzljak L, Gutierrez A, de la Calle A, Goldman-Rakic PS (1998). Prominence of the dopamine D2 short isoform in dopaminergic pathways. Proc Natl Acad Sci USA 95: 7731-7736.

Kilford EJ, Dumontheil I, Wood NW, Blakemore SJ (2014). Influence of COMT genotype and affective distractors on the processing of self-generated thought. Soc Cogn Affect Neurosci 10: 777-782.

Kohno M, Ghahremani DG, Morales AM, Robertson CL, Ishibashi K, Morgan AT et al (2013). Risk-taking behavior: dopamine D2/D3 receptors, feedback, and frontolimbic activity. Cereb Cortex 25: 236-245.

Kohno M, Morales AM, Ghahremani DG, Hellemann G, London ED (2014). Risky decision making, prefrontal cortex, and mesocorticolimbic functional connectivity in methamphetamine dependence. JAMA Psychiatry 71: 812-820.

Krain AL, Wilson AM, Arbuckle R, Castellanos FX, Milham MP (2006). Distinct neural mechanisms of risk and ambiguity: a meta-analysis of decision-making. NeuroImage 32: 477-484.

Lancaster TM, Linden DE, Heerey EA (2012). COMT val158met predicts reward responsiveness in humans. Genes Brain Behav 11: 986-992.

Lauzon NM, Laviolette SR (2010). Dopamine D4-receptor modulation of cortical neuronal network activity and emotional processing: implications for neuropsychiatric disorders. Behav Brain Res 208: 12-22.

Le Foll B, Guranda M, Wilson AA, Houle S, Rusjan PM, Wing VC et al (2013). Elevation of dopamine induced by cigarette smoking: novel insights from a [11C]-+-PHNO PET study in humans. Neuropsychopharmacology 39: 415-424.

Levine MS, Cepeda C (1998). Dopamine modulation of responses mediated by excitatory amino acids in the neostriatum. $A d v$ Pharmacol 42: 724-729.

Lippert RN, Ellacott KL, Cone RD (2014). Gender-specific roles for the melanocortin-3 receptor in the regulation of the mesolimbic dopamine system in mice. Endocrinology 155: 1718-1727.

Mata R, Hau R, Papassotiropoulos A, Hertwig R (2012). DAT1 polymorphism is associated with risk taking in the Balloon Analogue Risk Task (BART). PLoS One 7: e39135.

McGeer PL, McGeer EG, Scherer U, Singh K (1977). A glutamatergic corticostriatal path? Brain Res 128: 369-373.

Meltzer HY (1980). Relevance of dopamine autoreceptors for psychiatry: preclinical and clinical studies. Schizophr Bull 6: 456-475.

Meyer-Lindenberg A, Kohn PD, Kolachana B, Kippenhan S, McInerney-Leo A, Nussbaum R et al (2005). Midbrain dopamine and prefrontal function in humans: interaction and modulation by COMT genotype. Nat Neurosci 8: 594-596.

Nikolova YS, Ferrell RE, Manuck SB, Hariri AR (2011). Multilocus genetic profile for dopamine signaling predicts ventral striatum reactivity. Neuropsychopharmacology 36: 1940-1947.

Rahman S, JS B, N Cardinal R, Rogers R, Robbins T (2001). Decision making and neuropsychiatry. Trends Cogn Sci 5: 271-277.

Rao H, Korczykowski M, Pluta J, Hoang A, Detre JA (2008). Neural correlates of voluntary and involuntary risk taking in the human brain: an fMRI Study of the Balloon Analog Risk Task (BART). NeuroImage 42: 902-910.

Riba J, Kramer UM, Heldmann M, Richter S, Munte TF (2008). Dopamine agonist increases risk taking but blunts reward-related brain activity. PLoS One 3: e2479.

Riccardi P, Zald D, Li R, Park S, Ansari MS, Dawant B et al (2006). Sex differences in amphetamine-induced displacement of [(18)F] fallypride in striatal and extrastriatal regions: a PET study. Am J Psychiatry 163: 1639-1641.

Rudnick G, Clark J (1993). From synapse to vesicle: the reuptake and storage of biogenic amine neurotransmitters. Biochim Biophys Acta 1144: 249-263.

Savitz J, Hodgkinson CA, Martin-Soelch C, Shen PH, Szczepanik J, Nugent A et al (2013). The functional DRD3 Ser9Gly polymorphism (rs6280) is pleiotropic, affecting reward as well as movement. PLoS One 8: e54108.

Shaikh S, Collier D, Kerwin RW, Pilowsky LS, Gill M, Xu WM et al (1993). Dopamine D4 receptor subtypes and response to clozapine. Lancet 341: 116.

Shiels K, Hawk LW Jr, Reynolds B, Mazzullo RJ, Rhodes JD, Pelham WE Jr et al (2009). Effects of methylphenidate on discounting of delayed rewards in attention deficit/hyperactivity disorder. Exp Clin Psychopharmacol 17: 291-301.

St Onge JR, Ahn S, Phillips AG, Floresco SB (2012). Dynamic fluctuations in dopamine efflux in the prefrontal cortex and nucleus accumbens during risk-based decision making. J Neurosci 32: $16880-16891$.

St Onge JR, Floresco SB (2009). Dopaminergic modulation of risk-based decision making. Neuropsychopharmacology 34: 681-697.

Staley JK, Krishnan-Sarin S, Zoghbi S, Tamagnan G, Fujita M, Seibyl JP et al (2001). Sex differences in [123I]beta-CIT SPECT measures of dopamine and serotonin transporter availability in healthy smokers and nonsmokers. Synapse 41: 275-284.

Stice E, Yokum S, Burger K, Epstein L, Smolen A (2012). Multilocus genetic composite reflecting dopamine signaling capacity predicts reward circuitry responsivity. J Neurosci 32: 10093-10100.

Stopper CM, Tse MT, Montes DR, Wiedman CR, Floresco SB (2014). Overriding phasic dopamine signals redirects action selection during risk/reward decision making. Neuron 84: 177-189.

Sugam JA, Day JJ, Wightman RM, Carelli RM (2012). Phasic nucleus accumbens dopamine encodes risk-based decisionmaking behavior. Biol Psychiatry 71: 199-205.

Usiello A, Baik JH, Rouge-Pont F, Picetti R, Dierich A, LeMeur M et al (2000). Distinct functions of the two isoforms of dopamine D2 receptors. Nature 408: 199-203.

Uzefovsky F, Shalev I, Israel S, Edelman S, Raz Y, Perach-Barzilay N et al (2014). The dopamine D4 receptor gene shows a gendersensitive association with cognitive empathy: evidence from two independent samples. Emotion 14: 712-721.

Van Tol HH, Wu CM, Guan HC, Ohara K, Bunzow JR, Civelli O et al (1992). Multiple dopamine D4 receptor variants in the human population. Nature 358: 149-152.

Volkow ND, Chang L, Wang G-J, Fowler JS, Ding Y-S, Sedler M et al (2001). Low level of brain dopamine $\mathrm{D}_{2}$ receptors in methamphetamine abusers: association with metabolism in the orbitofrontal cortex. Am J Psychiatry 158: 2015-2021.

Wise RA (2002). Brain reward circuitry: insights from unsensed incentives. Neuron 36: 229-240.

Zack M, Poulos CX (2004). Amphetamine primes motivation to gamble and gambling-related semantic networks in problem gamblers. Neuropsychopharmacology 29: 195-207.

Zack M, Poulos CX (2007). A D2 antagonist enhances the rewarding and priming effects of a gambling episode in pathological gamblers. Neuropsychopharmacology 32: 1678-1686.

Supplementary Information accompanies the paper on the Neuropsychopharmacology website (http://www.nature.com/npp) 